\title{
Constrained variational calculations for finite nuclei
}

\author{
R F Bishop†, C Howes, J M Irvine and M Modarres \\ Department of Theoretical Physics, The University, Manchester M13 9PL, UK and \\ Science Research Council, Daresbury Laboratory, Daresbury, Warrington WA4 4AD, \\ UK
}

Received 25 May 1978

\begin{abstract}
The technique of lowest-order constrained variational calculations used by us to study the bulk properties of homogeneous, quantal fluids is extended for use in discussing the ground-state properties of finite nuclei.

The significance of various constraints in finite systems is discussed. It is shown that the saturating effects of explicit allowance for $\mathrm{N}^{*}(1234)$ excitations is essential if the calculations are to yield sensible binding energies and RMs radii.

Specific application is made to the light closed-shell nuclei ${ }^{4} \mathrm{He},{ }^{12} \mathrm{C}$ and ${ }^{16} \mathrm{O}$.
\end{abstract}

\section{Introduction}

In a series of papers Owen et al (1976a, b, 1977) have developed a technique of lowest-order constrained variational (LOCV) calculation to study the bulk properties of quantal fluids. The technique was extended by Howes et al (1978a) to calculate the symmetry coefficient for the semi-empirical mass formula from asymmetric nucleon matter and eventually to a study of the properties of beta-stable matter (Howes et al 1978b). Work is at present in progress on a study of the surface energies of quantal fluids using developments of the same technique (Howes et al 1978c).

We have considered systems described by a Hamiltonian $H$ of the form

$$
H=-\frac{\hbar^{2}}{2 m} \sum_{i=1}^{A} \nabla_{i}^{2}+\sum_{i<j=1}^{A} V(i j) \quad A \rightarrow \infty
$$

and a trial variational wavefunction of the form

$$
\Psi=\mathscr{P}\left(\prod_{i<j} f(i j) \prod_{i} \phi_{i}\left(\boldsymbol{r}_{i}\right)\right)
$$

where the operator $\mathscr{P}$ either symmetrises or antisymmetrises the product wavefunction depending on whether we are dealing with a Bose or Fermi system. For an homogeneous Fermi system the particle number density $n^{(1)}$ is given in terms of the Fermi wavenumber $k_{\mathrm{F}}$ :

$$
n^{(1)}=A / V=v k_{\mathrm{F}}^{3} / 6 \pi^{2}
$$

† Present address: Nuclear Science Division, Lawrence Berkeley Laboratory, Berkeley, California 94720 , USA. 
where $v$ is the spin-isospin degeneracy factor, e.g. 4 and 2 for nuclear matter and neutron matter respectively, and the uncorrelated single-particle states

$$
\phi_{i}\left(r_{i}\right)=V^{-1 / 2} \exp \left(\mathrm{i} k_{i} \cdot r_{i}\right) \gamma_{i}(i) \quad k_{i}<k_{\mathrm{F}}
$$

are normalised in a volume $V$.

The trial energy functional $E([f],[\phi])$

$$
E([f],[\phi])=\frac{\left\langle\Psi^{*}|H| \Psi\right\rangle}{\left\langle\Psi^{*} \mid \Psi\right\rangle}
$$

may then be expanded in a cluster series

$$
E=E_{1}+E_{2}+\ldots
$$

where

$$
E_{1}=\sum_{i=1}^{A}\left(\phi_{i}^{*}\left|-\frac{\hbar^{2}}{2 m} \nabla^{2}\right| \phi_{i}\right)=\left(3 \hbar^{2} k_{\mathrm{F}}^{2} / 10 m\right) A
$$

and

$$
\begin{aligned}
& E_{2}=\sum_{i>j=1}^{A}\left(\phi_{i} \phi_{j}|\mathscr{V}| \phi_{i} \phi_{j}-\phi_{j} \phi_{i}\right) \\
& \mathscr{V}(12)=-\frac{\hbar^{2}}{2 m}\left[f(12),\left[\nabla_{12}^{2}, f(12)\right]\right]+f(12) V(12) f(12) .
\end{aligned}
$$

We have considered two-body potentials having the general form

$$
V(12)=\sum_{\alpha i} V_{\alpha}(12) P_{\alpha}^{(i)}
$$

where $P_{\alpha}^{(i)}$ is the $\alpha$-channel projection operator and the superscript labels different orthogonal coupled-channel components. In each channel we have assumed we may write the potential in the form

$$
V_{\alpha}(12)=V_{\alpha}^{\mathrm{c}}\left(r_{12}\right)+V_{\alpha}^{\mathrm{T}}\left(r_{12}\right) S_{12}+V_{\alpha}^{L S}\left(r_{12}\right) L . S
$$

where $S_{12}$ and $L . S$ are the usual tensor and spin-orbit operators respectively. In all channels except the spin-triplet states with $L=J \pm 1$ the tensor and spin-orbit components may be combined with the central potential to form an effective central potential which we continue to denote by $V_{x i}^{\mathrm{c}}(\alpha=J, L, S, T, i=1)$ and where the need for the additional label $i$ will become obvious when we consider the $S=1$, $L=J \pm 1$ channels. Corresponding to the potential given in equation (10), we assume

$$
f(12)=\sum_{\alpha i} f_{\alpha}^{(i)}(12) P_{\alpha}^{(i)}
$$

where we limit $f^{(i)}(12)$ to reproduce central and tensor correlations. With tensor correlations the channels $\alpha=J(=L+1), L, S(=1), T$ and $\alpha=J(=L-1), L, S(=1)$, $T$ are coupled and we choose to work in an orthogonal channel basis projected by the operators (Irvine et al 1976, Owen et al 1976b, Irvine et al 1976)

$$
P_{\alpha}^{(2)}=\frac{2}{3}+\frac{1}{6} S_{12} \quad \text { and } \quad P_{\alpha}^{(3)}=1-P_{\alpha}^{(2)}=\frac{1}{3}-\frac{1}{6} S_{12} .
$$

In this basis we have the boundary condition

$$
f_{\alpha}^{(i)}(12) \rightarrow 1 \quad \text { as } r_{12} \rightarrow \infty \quad(\text { all } \alpha \text { and } i) .
$$


Including tensor correlations means that the correlation operators $f(12)$ no longer commute and leads to a considerable complication in the higher cluster terms in the expansion in equation (6). These complications have severely hindered progress in the programmes based upon the fermion hypernetted chain (FHNC) and MonteCarlo (MC) approaches to the study of dense Fermi systems. In the works of Owen et al we have described a method of LOCV which involves truncation of the cluster series (equation (6)) at the two-body term, but with the imposition of constraints on (the channel components of the two-body correlation function $f(12)$ which are devised to justify this truncation. We have shown that our LoCv calculation gives excellent agreement with the FHNC and MC calculations for the Bethe 'homework' problem with central potentials (Owen et al 1975, 1976a) and we have published the results of LOCV calculations for the new 'homework' problem for non-central forces (Howes et al 1978d) and look forward to the appearance of FHNC and MC results with which to compare our calculations.

For constraints formulated so that $f_{x}^{(i)}(r)$ satisfies a bound of the form

$$
0 \leqslant f_{x}^{(i)}(r) \leqslant h_{x}^{(i)}(r)
$$

and simultaneously an integral inequality of the form

$$
\sum_{x, i} 4 \pi n^{(1)} \int_{0}^{\infty} G_{\alpha}^{(i)}\left[f_{\alpha}^{(i)}(r), h_{\alpha}^{(i)}(r)\right] \mathrm{d} r \leqslant I
$$

where the functional $G_{z}^{(i)}$ satisfies the condition

$$
G_{x}^{(i)}\left[h_{x}^{(i)}(r), h_{x}^{(i)}(r)\right] \equiv 0,
$$

the Locv Euler-Lagrange equations are

$$
\begin{aligned}
& g_{x}^{(1)^{\prime \prime}}(r)-\left(\frac{a_{x}^{(1)^{\prime \prime}}}{a_{\alpha}^{(1)}}+\frac{m}{\hbar^{2}} V_{\alpha}^{\mathrm{c}}\right) g_{x}^{(1)}(r)+\frac{m \lambda}{2 \hbar^{2} a_{x}^{(1)}(r)} \frac{\delta G_{\alpha}^{(1)}}{\delta f_{x}^{(1)}}=0 \\
& g_{\alpha}^{(2)^{\prime \prime}}(r)-\left(\frac{a_{\alpha}^{(2)^{\prime \prime}}}{a_{\alpha}^{(2)}}+\frac{m}{\hbar^{2}}\left(V_{\alpha}^{\mathrm{c}}+2 V_{\alpha}^{\mathrm{T}}-V_{\alpha}^{L S}\right)+\frac{b_{\alpha}^{2}}{r^{2} a_{\alpha}^{(2) 2}}\right) g_{\alpha}^{(2)}(r) \\
& +\left[\left(\frac{1}{r^{2}}-\frac{m}{2 \hbar^{2}} V_{\alpha}^{L S}\right) \frac{b_{\alpha}^{2}}{\left(a_{x}^{(2)} a_{\alpha}^{(3)}\right)}\right] g_{\alpha}^{(3)}(r)+\frac{m \lambda}{2 \hbar^{2} a_{x}^{(2)}} \frac{\delta G_{\alpha}^{(2)}}{\delta f_{\alpha}^{(2)}}=0 \\
& g_{\alpha}^{(3)^{\prime \prime}}(r)-\left(\frac{a_{\alpha}^{(3)^{\prime \prime}}}{a_{\alpha}^{(3)}}+\frac{m}{\hbar^{2}}\left(V_{\alpha}^{\mathrm{c}}-4 V_{\alpha}^{\mathrm{T}}-2 V_{\alpha}^{L S}\right)+\frac{b_{\alpha}^{2}}{r^{2} a_{\alpha}^{(3) 2}}\right) g_{\alpha}^{(3)}(r) \\
& +\left[\left(\frac{1}{r^{2}}-\frac{m}{2 \hbar^{2}} V_{\alpha}^{L S}\right) \frac{b_{\alpha}^{2}}{\left(a_{x}^{(2)} a_{x}^{(3)}\right)}\right] g_{\alpha}^{(2)}(r)+\frac{m \lambda}{2 \hbar^{2} a_{x}^{(3)}} \frac{\delta G^{(3)}}{\delta f_{x}^{(3)}}=0
\end{aligned}
$$

where $\lambda$ is the Lagrange multiplier associated with the constraint (15) and primes denote differentiation with respect to $r$. In equations (17)

with

$$
g_{x}^{(i)} \equiv a_{x}^{(i)}\left(k_{\mathrm{F}} r\right) f_{x}^{(i)}(r)
$$

$$
\begin{aligned}
& a_{x}^{(1) 2}(x) \equiv x^{2} I_{L}(x) \\
& a_{\alpha}^{(2) 2}(x) \equiv x^{2}(2 J+1)^{-1}\left[(J+1) I_{J-1}(x)+J I_{J+1}(x)\right] \\
& a_{\alpha}^{(3) 2}(x) \equiv x^{2}(2 J+1)^{-1}\left[\left(J I_{J-1}(x)+(J+1) I_{J+1}(x)\right]\right. \\
& b_{\alpha}^{2}(x) \equiv x^{2} 2 J(J+1)(2 J+1)^{-1}\left(I_{J-1}(x)-I_{J+1}(x)\right)
\end{aligned}
$$


and

$$
I_{L}(x) \equiv 48 \int_{0}^{1} \mathrm{~d} z z^{2}\left(1-\frac{3}{2} z+\frac{1}{2} z^{3}\right) j_{L}^{2}(x z),
$$

$j_{L}$ being the usual spherical Bessel function. The form of the functions $a_{\alpha}^{(i)}$ and $b_{\alpha}$ follows from the plane-wave nature of the single-particle states $\phi_{i}$ and the sum (integral) over the occupied states of the Fermi sea. In the following section we shall begin our discussion of how the Euler-Lagrange equations must be modified for a study of finite nuclei.

\section{Cluster energies for finite nuclei}

Ideally the single-particle wavefunctions should be determined variationally subject to the constraints $\left(\phi_{i} \mid \phi_{j}\right)=\delta_{i j}$, i.e.

$$
\frac{\delta E}{\delta \phi_{i}}-\epsilon_{i} \phi_{i}=0
$$

where the 'single-particle energies' $\epsilon_{i}$ are the Lagrange multipliers of the orthonormality constraints. Inserting the truncated expansion (6), equations (21) become a set of 'Hartree-Fock' equations with the 'effective' two-body interaction $\mathscr{V}(12)$ of equation (8). In this preliminary investigation of light nuclei we shall not solve equations (21) but simply assume that the $\phi_{i}$ may be approximated by oscillator functions leaving the oscillator energy $\hbar \omega$ as a single variational parameter.

We are now describing localised finite nuclei and have lost the translational invariance which characterised the nucleon fluid calculations. We define the origin of our coordinate system to be the centre of mass of our nucleus, i.e.

$$
\sum_{i=1}^{A} \boldsymbol{r}_{i}=0
$$

and thus lose a degree of freedom. Hence it becomes more appropriate to consider the intrinsic Hamiltonian (Irvine et al 1976)

$$
\begin{aligned}
H_{0} & =H-P^{2} / 2 M \\
& =\sum_{i j}\left(\frac{p_{i j}^{2}}{M}+V(i j)\right)
\end{aligned}
$$

where $P$ is the centre-of-mass momentum and $M$ is the total mass:

$$
\boldsymbol{P}=\sum_{i=1}^{A} p_{i} \quad M=A m .
$$

In dealing with finite nuclei we shall throughout define the relative and centre-of-mass coordinates of a pair of particles by

$$
\boldsymbol{r}_{i j}=\left(\boldsymbol{r}_{i}-\boldsymbol{r}_{j}\right) / \sqrt{2} \quad \boldsymbol{R}_{i j}=\left(\boldsymbol{r}_{i}+\boldsymbol{r}_{j}\right) / \sqrt{2}
$$

and the corresponding conjugate momenta. Our intrinsic Hamiltonian $H_{0}$ has no one-body part and hence in our cluster expansion $E_{1}=0$ while $E_{2}$ is of the form given in equation (8) but with $\mathscr{N}(12)$ simply given by

$$
\mathscr{V}(12)=f(12)\left(-\frac{\hbar^{2}}{A m} \nabla_{12}^{2}+V(12)\right) f(12)
$$


and the sum over states running over a finite set of occupied oscillator orbitals. The two-body oscillator equations can be decomposed into relative and centre-ofmass coordinates using the familiar Brody-Moshinsky (1960) transformation brackets $\left\langle n l, N L, \lambda \mid n_{1} l_{1}, n_{2} l_{2}, \hat{\lambda}\right\rangle$ and the operator $\mathscr{V}(12)$ acts on the relative coordinate function. For a closed-shell $N=Z$ nucleus the two-body cluster energy can then be written

$$
\begin{aligned}
E_{2}=\sum_{i \leqslant j}(1+ & \left.\delta_{i j}\right)^{-1}\left[j_{i}\right]\left[j_{j}\right][\lambda]\left[\lambda^{\prime}\right][S][j][J][T](-1)^{\lambda+\lambda^{\prime}}\left\{\begin{array}{lll}
L & l^{\prime} & \lambda^{\prime} \\
S & j & J
\end{array}\right\}\left\{\begin{array}{lll}
L & l & \lambda \\
S & j & J
\end{array}\right\} \\
& \times\left\{\begin{array}{lll}
l_{i} & \frac{1}{2} & j_{i} \\
l_{j} & \frac{1}{2} & j_{j} \\
\lambda & S & j
\end{array}\right\}\left\{\begin{array}{lll}
l_{i} & \frac{1}{2} & j_{i} \\
l_{j} & \frac{1}{2} & j_{j} \\
\lambda^{\prime} & S & j
\end{array}\right\}\left\langle n l, N L, \lambda \mid n_{i} l_{i}, n_{j} l_{j}, \lambda\right\rangle\left\langle n^{\prime} l^{\prime} N L \lambda^{\prime} \mid n_{i} l_{i} n_{j} l_{j} \lambda^{\prime}\right\rangle \\
& \times\left[1-(-1)^{l+S+T}\right]\left\langle n^{\prime}\left(l^{\prime} S\right) J T|\mathscr{V}| n(l S) J T\right\rangle
\end{aligned}
$$

with the usual notation $[J]=2 J+1$ etc. The sum on the right-hand side of equation (27) is over all labels with appropriate limits set by the sums on $i\left(=n_{i}, l_{i}, j_{i}\right)$ and $j$ over the occupied orbitals of the relevant nucleus.

\section{Constraints for finite nuclei}

Quantal systems of identical particles contain correlations induced by the appropriate statistics. For example, in an infinite Bose system with all the particles in the zeromomentum condensate the non-interacting relative two-body wavefunction is given by

$$
r^{2} \phi_{\mathrm{B}}^{2}(r)=r^{2}(1-1 / A)
$$

while for a degenerate system of fermions averaging over the Fermi sea yields the non-interacting fermion wavefunction

$$
r^{2} \phi_{\mathrm{F}}^{2}(r)=r^{2}\left(1-\frac{9}{v} \frac{j_{1}^{2}\left(k_{\mathrm{F}} r\right)}{\left(k_{\mathrm{F}} r\right)^{2}}\right) .
$$

Switching on an interaction induces further two-body correlations described by the functions $f(12)$.

For infinite homogeneous systems we have considered two sets of conditions for equations (14), (15) and (16). In both cases we have assumed $h_{\alpha}^{(i)}(r)$ to be independent of the channel. We shall continue to use this assumption in the present work although the sensitivity of our results to this assumption is being investigated. The first set of conditions is given by

$$
G[f, h]=\left(h^{2}(r)-f^{2}(r)\right) r^{2} \phi^{2}
$$

with

$$
h(r)=1 \quad \text { and } \quad I=1 .
$$

With this choice the constraint (15) is that originally introduced by Pandharipande $(1971,1972)$ and has the physical interpretation that on average the wound induced in the wavefunction around an average particle in the system excludes less than one other particle, making the convergence of the cluster expansion a plausible 
assumption. This physical interpretation is clearly less obvious in an inhomogeneous system like a finite nucleus. For an infinite Bose system we see that to $\mathrm{O}(1 / A)$

$$
G=\left(1-f^{2}(r) \phi^{2}\right) r^{2}
$$

and identifying $f^{2} \phi^{2}$ as the two-body approximation to the two-body radial distribution function the integral of (15) taken with the equality merely expresses the normalisation condition which must be satisfied by any exact radial distribution function. It is known (Mueller and Clark 1970, Ristig et al 1971) that the imposition of the normalisation condition has the effect of stimulating rapid convergence of the cluster expansion. We have adopted the convention of calling the choice (31) the Bose constraint. The analogous Fermi normalisation constraint condition is then

$$
h(r)=\left[1-\frac{9}{v}\left(\frac{j_{1}\left(k_{\mathrm{F}} r\right)}{k_{\mathrm{F}} r}\right)^{2}\right]^{-1 / 2} \quad \text { and } \quad I=1 .
$$

For the homogenous Fermi fluids we have integrated the Euler-Lagrange equations (17) out from the origin where we have the boundary condition

$$
f_{x}^{(i)}(r=0)=0
$$

until the point $d^{(i)}$ where the logarithmic derivative of $f_{\alpha}^{(i)}(r)$ equals the logarithmic derivative of $h(r)$ and for $r \geqslant d_{\alpha}^{(i)}$ we have used $f_{\alpha}^{(i)}\left(r>d_{\alpha}^{(i)}\right) \equiv h(r)$.

For finite nuclei we again consider the normalisation of the two-body density function, i.e. for the uncorrelated system

$$
\frac{1}{2} \int n_{0}^{(2)}\left(\boldsymbol{r}_{1}, \boldsymbol{r}_{2}\right) \mathrm{d} \boldsymbol{r}_{1} \mathrm{~d} \boldsymbol{r}_{2}=\sum_{i>j} \int\left|\Phi_{i j}^{(2)}\left(\boldsymbol{r}_{1}, \boldsymbol{r}_{2}\right)\right|^{2} \mathrm{~d} \boldsymbol{r}_{1} \mathrm{~d} \boldsymbol{r}_{2}=\frac{1}{2} A(A-1)
$$

where $\Phi_{i j}^{(2)}\left(r_{1}, r_{2}\right)$ is the non-interacting two-body wavefunction

$$
\Phi_{i j}^{(2)}=\frac{1}{\sqrt{2}}\left(\phi_{i}\left(\boldsymbol{r}_{1}\right) \phi_{j}\left(\boldsymbol{r}_{2}\right)-\phi_{i}\left(\boldsymbol{r}_{2}\right) \phi_{j}\left(\boldsymbol{r}_{1}\right)\right) .
$$

For the homogeneous fluids the single-particle functions $\phi_{i}(r)$ are plane waves, the sums $\Sigma_{i>j}$ are integrals over the Fermi sea and $n_{0}^{(2)} \equiv n^{(1) 2} h^{-2}(r)$ of equation (35). For the finite nuclei the $\phi_{i}$ will be harmonic oscillator functions and the sums are over occupied orbitals for the relevant nucleus. The correlated two-body functions also satisfied a normalisation condition

$$
\begin{aligned}
\frac{1}{2} \int n^{(2)}\left(\boldsymbol{r}_{1}, \boldsymbol{r}_{2}\right) \mathrm{d} \boldsymbol{r}_{1} \mathrm{~d} \boldsymbol{r}_{2} & =\sum_{i>j} \int\left|\Psi_{i j}^{(2)}\left(\boldsymbol{r}_{1}, \boldsymbol{r}_{2}\right)\right|^{2} \mathrm{~d} \boldsymbol{r}_{1} \mathrm{~d} \boldsymbol{r}_{2} \\
& =\sum_{i>j}\left\langle\Phi_{i j}^{(2)}\left|f^{2}\right| \Phi_{i j}^{(2)}\right\rangle=\frac{1}{2} A(A-1) .
\end{aligned}
$$

Subtracting equation (37) from equation (35) we have

$$
\sum_{i>j} \mid\left\langle\Phi_{i j}^{(2)}\left|1-f^{2}\right| \Phi_{i j}^{(2)}\right\rangle=0
$$

It is quite clear that equation (38) can only be satisfied if $f(r)$ overshoots unity as it does in the case of our Fermi normalisation constraint.

We note that the expression on the left-hand side of equation (38) is one of the smallness parameters $k$ used to judge the convergence of the cluster expansion 
(Owen et al 1976a). We thus consider as an approximation to equation (38) the constraint

$$
\sum_{i>j} \mid\left\langle\Phi_{i j}^{(2)}\left|1-f^{2}\right| \Phi_{i j}^{(2)}\right\rangle \leqslant k
$$

where for $f \leqslant 1$ this is the natural extension of the Bose constraint to the finite nucleus. We seek the smallest value of $k$ for which we can find solutions to the Euler-Lagrange equations. If, as we shall find, $k$ is of order unity we see that this implies that the excluded volume is of $\mathrm{O}(1 / A)$. We shall continue to call these conditions the Bose constraint.

In general, in an inhomogeneous system the two-body correlation function $f$ will not only be a function of the relative coordinate $r$ of the two particles but may also depend on the centre-of-mass coordinate $R$ of the pair. For the $N=Z$ light closed-shell nuclei, assuming unperturbed oscillator single-particle functions, the function analogous to $h(r)$ in equation (33) is

$$
\hbar(r, R)=\left(1-\frac{1}{4} \frac{\left[1+b^{-2} \alpha^{2}\left(R^{2}-r^{2}\right)\right]^{2}}{1+2 b^{-2} \alpha^{2}\left(R^{2}+r^{2}\right)+b^{-4} \alpha^{4}\left(R^{2}-r^{2}\right)}\right)^{-1 / 2}
$$

where for ${ }^{4} \mathrm{He},{ }^{12} \mathrm{C}$ and ${ }^{16} \mathrm{O}, \alpha$ is 0,2 and 3 respectively and $b$ is the oscillator size parameter. Alternatively we could use a local density approximation with $f$ healing to

$$
h_{\mathrm{LD}}(r, R)=\left[1-\frac{9}{4}\left(\frac{j_{1}\left(k_{\mathrm{F}}(R) r\right)}{k_{\mathrm{F}}(R) r}\right)^{2}\right]^{-1 / 2}
$$

where we use equation (3) to define a local $k_{\mathrm{F}}(R)$ depending on the density $n^{(1)}(R)$ at the centre of mass of the correlated pair of particles. The consequences of these various prescriptions for the healing properties of the correlation function in finite systems are being studied. In the present work we shall make the restriction that all channel correlation functions $f_{\alpha}^{(i)}$ heal to the same function

$$
h_{\mathrm{efr}}(r, \bar{k})=\left[1-\frac{9}{4}\left(\frac{j_{1}(\bar{k} r)}{\bar{k} r}\right)^{2}\right]^{-1 / 2}
$$

and we shall investigate how our results depend on the parameter $\vec{k}$. This is the natural generalisation of our normalisation condition to finite nuclei and we shall solve the Euler--Lagrange equations subject to the constraint of equation (38) with the boundary condition

$$
f_{\alpha}^{(i)}(r) \underset{r \rightarrow \infty}{\longrightarrow} h_{\mathrm{eff}}(r, \bar{k})
$$

\section{The saturating effect of the $N^{*}(1234)$}

In our analysis of nuclear matter with realistic potentials (Owen et al 1977) we found that our LOCV calculations yielded saturation with binding energies of 20 to $23 \mathrm{MeV} / \mathrm{N}$ at densities corresponding to $k_{\mathrm{F}} \simeq 1.7 \mathrm{fm}^{-1}$. We found that the explicit inclusion of the possibility of excitation of nucleons to $\mathrm{N}^{*}(1234)$ 's improved significantly the nuclear matter saturation prediction to binding energies $\sim 16 \mathrm{MeV} / \mathrm{N}$ at $k_{\mathrm{F}} \sim 1.4 \mathrm{fm}^{-1}$. Similarly Howes et al (1978a) found that the explicit inclusion of 
the $N^{*}(1234)$ led to an improved prediction of the symmetry coefficient in the semiempirical mass formula in asymmetric nucleon fluids. If these results are translated into finite nucleus calculations we would expect the results of our LOCV calculations with the Reid potential to significantly overbind the nuclei with RMS radii which were much smaller than those observed and that an explicit allowance for the $N^{*}$ (1234) excitations would go some way towards rectifying this. In the work of Modarres et al (1978) the saturating effect of the $N^{*}(1234)$ in light nuclei has been studied.

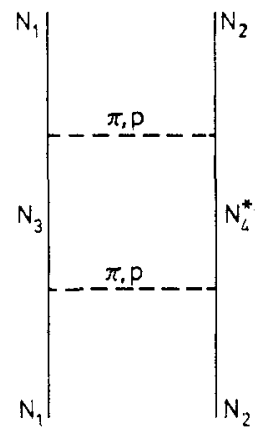

Figure 1. A two-boson exchange contribution to the $\mathrm{N}-\mathrm{N}$ interaction.

The amplitude in figure 1 presumably contributes to the total $\mathrm{N}-\mathrm{N}$ interaction. When this occurs inside the nucleus there are two effects to be allowed for: first, the intermediate nucleon is excluded from occupied nucleon states (Pauli effect) and, second, the intermediate nucleon and $N^{*}$ experience the mean field of the other nucleons (dispersion effect). This leads to energy shifts $\Delta E(\mathrm{P})$ and $\Delta E(\mathrm{D})$ respectively in finite nuclei, where

$$
\begin{gathered}
\Delta E(\mathrm{P})=\frac{1}{2} \sum_{\substack{\mathrm{N}_{1} \mathrm{~N}_{2} \mathrm{~N}_{3} \\
\mathrm{~N}_{4}^{*}}}\left|\left\langle\mathrm{~N}_{1} \mathrm{~N}_{2}\left|V_{\mathrm{NN}^{*}}\right| \mathrm{N}_{3} \mathrm{~N}_{4}^{*}\right\rangle\right|^{2}\left(E_{4}^{*}+E_{3}-E_{1}-E_{2}\right)^{-1} \\
\Delta E(\mathrm{D})=\frac{1}{2} \sum_{\substack{\mathrm{N}_{1} \mathrm{~N}_{2} \\
\mathrm{~N}_{3} \mathrm{~N}_{4}^{*}}}\left|\left\langle\mathrm{~N}_{1} \mathrm{~N}_{2}\left|V_{\mathrm{NN}^{*}}\right| \mathrm{N}_{3} \mathrm{~N}_{4}^{*}\right\rangle\right|^{2}\left[\left(T_{4}^{*}+T_{3}-T_{1}-T_{2}\right)^{-1}\right. \\
\left.-\left(E_{4}^{*}+E_{3}-E_{1}-E_{2}\right)^{-1}\right]
\end{gathered}
$$

with $V_{\mathrm{NN}^{*}}$ the one-pion and one-rho exchange transition potential used by Modarres et al (1978)† and where the single-nucleon energies

$$
E_{i}=T_{i}+U_{i}
$$

are composed of a kinetic energy $T_{i}$ and a mean-field potential energy $U_{i}$. The corresponding $\mathrm{N}^{*}$ energies are

$$
T_{4}^{*}=T_{4}+\Delta
$$

and

$$
E_{4}^{*}=E_{4}+\Delta
$$

$\uparrow$ Note that there is a misprint in the definition of $V_{\mathrm{N}} \mathrm{*}^{*}$ in the Letter by Modarres et al. The potential defined in equation (14) of that Letter should contain a factor $[1-\exp (-\lambda r)]^{2}$. 
where $\Delta=m_{\mathrm{N}^{*}}-m_{\mathrm{N}} \simeq 300 \mathrm{MeV}$. The summation over nucleon states $\mathrm{N}_{i}(i=1,2,3)$ in equation (44) is restricted to occupied nucleon orbitals in the nucleus under study while the resonance state $\mathrm{N}_{4}^{*}$ is summed over a complete set of states. In the dispersion term the nucleon states $N_{i}(i=1,2)$ are restricted to occupied orbitals and the states $\mathrm{N}_{3}$ and $\mathrm{N}_{4}^{*}$ are taken over a complete set of states. Using an oscillator approximation for the single-particle states we have

$$
T_{i}=\frac{1}{2}\left(2 n_{i}+l_{i}+\frac{3}{2}\right) \hbar \omega
$$

and

$$
U_{i}=\frac{1}{2}\left(2 n_{i}+l_{i}+\frac{3}{2}\right) h \omega-U_{0}
$$

for the nucleons, while for the $\mathrm{N}^{*}$ we assume that $U_{i}^{*}=U_{i}$ but that the kinetic energy has the form (48) but with the oscillator frequency

$$
\omega^{*}=\left(m_{\mathrm{N}} / m_{\mathrm{N}^{*}}\right) \omega=0.758 \omega .
$$

We can thus obtain for any light nucleus a total saturating effect from the explicit allowance for the $\mathrm{N}^{*}$

$$
\Delta E_{\mathrm{N}^{*}}\left(\hbar \omega, U_{0}\right)=\Delta E(\mathrm{P})+\Delta E(\mathrm{D})
$$

which is a function of the oscillator frequency, which in turn determines the RMS radius, and the depth of the mean field $U_{0}$. Results for the nuclei ${ }^{4} \mathrm{He},{ }^{12} \mathrm{C}$ and ${ }^{16} \mathrm{O}$ are given by Modarres et al (1978).

Strictly speaking, our $\Delta E_{\mathrm{N}^{*}}$ should be added to our two-body cluster energy (equation (8)) and the constrained variation carried out on the result (note that $\Delta E_{\mathrm{N}^{*}}$ depends on the two-body correlation function through the correlated two-nucleon state $\Psi_{\mathrm{N}_{1} N_{2}}^{(2)}$ in equations (44) and (45)). In the present work we have carried out our LOCV calculation to obtain a binding energy against harmonic oscillator energy curve $E_{\mathrm{LOCV}}(\hbar \omega, K)$ where $K=k$ for the Bose constraint (equation (39)) and $K=\bar{k}$ for the normalisation constraint (equation (42)). To this we have added $\Delta E_{\mathrm{N}^{*}}$ to obtain our final predicted equation of state

$$
E\left(\hbar \omega, K, U_{i}\right)=E_{\mathrm{LOCV}}+\Delta E_{\mathrm{N}^{*}}
$$

The only measure of 'self-consistency' that we have required is that $U$ be given by

$$
\begin{aligned}
E_{\mathrm{LOCv}} & =\left\langle\frac{1}{2} \sum_{i=1}^{A}\left(T_{i}+E_{i}\right)-\frac{P^{2}}{2 M}\right\rangle \\
& =\left(3 \sum_{i}\left(2 l_{i}+1\right)\left(2 n_{i}+l_{i}+\frac{3}{2}\right) \hbar \omega\right)-\frac{1}{2} A U_{0}-\frac{3}{2} \hbar \omega .
\end{aligned}
$$

Thus we have a single variational parameter $h \omega$ and a single constraint parameter $K$. Modarres et al (1978) have shown that the very rapid increase of $\Delta E_{\mathrm{N}^{*}}$ with increasing $\hbar \omega$ will help saturation in the finite nuclei, this effect coming principally from $\Delta E(\mathrm{P})$. Using equation (53) to define $U_{0}$ will also aid saturation since as the LOCV estimate of the binding increases, so will $U_{0}$, and this in turn leads to a sharp increase in the dispersion effect energy $\Delta E(\mathrm{D})$. 


\section{Results}

\subsection{The Bose constraint}

In figures 2, 3 and 4 we present the results of LOCV calculations using the Bose constraint for the nuclei ${ }^{4} \mathrm{He},{ }^{12} \mathrm{C}$ and ${ }^{16} \mathrm{O}$ respectively. We have considered a number of values of the constraint parameter $k$. We were unable to find stable solutions to our Euler-Lagrange equations if this parameter was too small $(k \leqslant 0.15)$. On the other hand, an LOCV calculation does not have much meaning if $k$ is too large $(k \geq 0.4)$. In all cases it was possible to choose a value of $k$ for which the LOCV

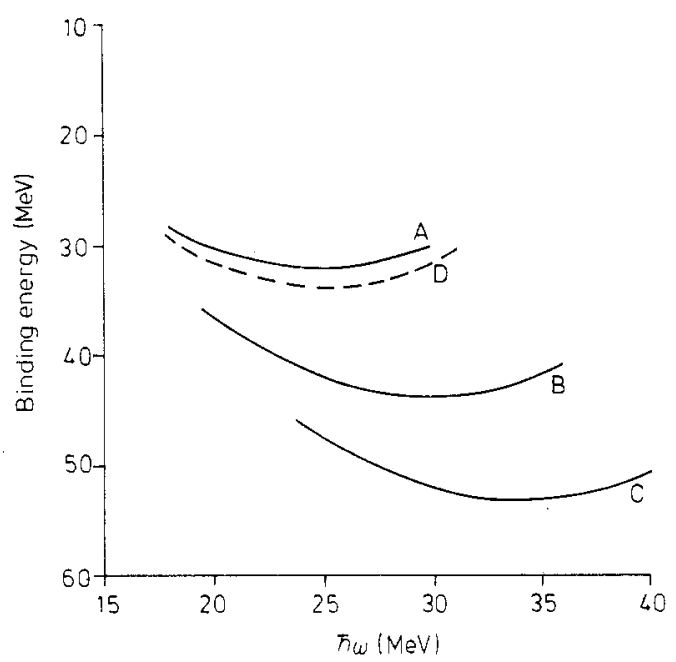

Figure 2. The full curves represent the results of Locv calculations using the Bose constraint with $k=(\mathrm{A}) 0.2$, (B) 0.3 and (C) 0.4 for ${ }^{4} \mathrm{He}$. The broken curve (D) is the result of including the explicit saturating effects of the $\mathrm{N}^{*}(1234)$ for $k=0.3$ which yields saturation close to the experimentally observed binding energy.

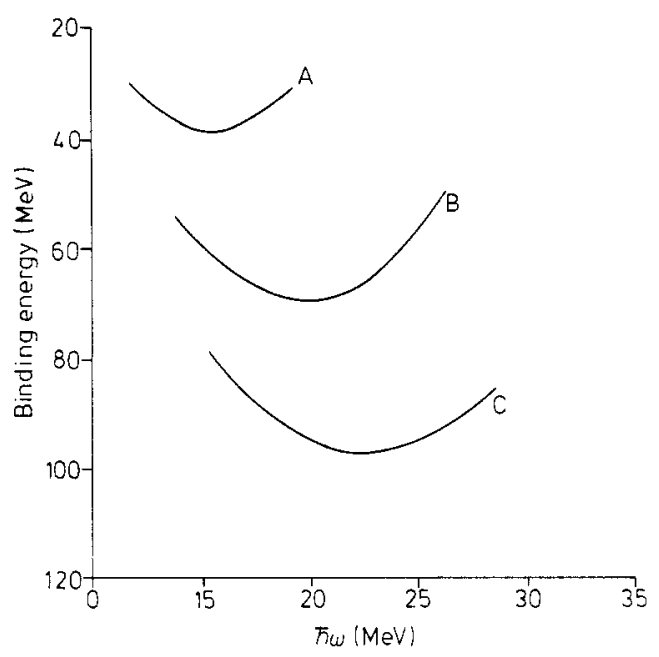

Figure 3. The results of Locv calculations with the Bose constraint with $k=(\mathrm{A}) 0 \cdot 2$, (B) 0.3 and (C) 0.4 for ${ }^{12} \mathrm{C}$. 


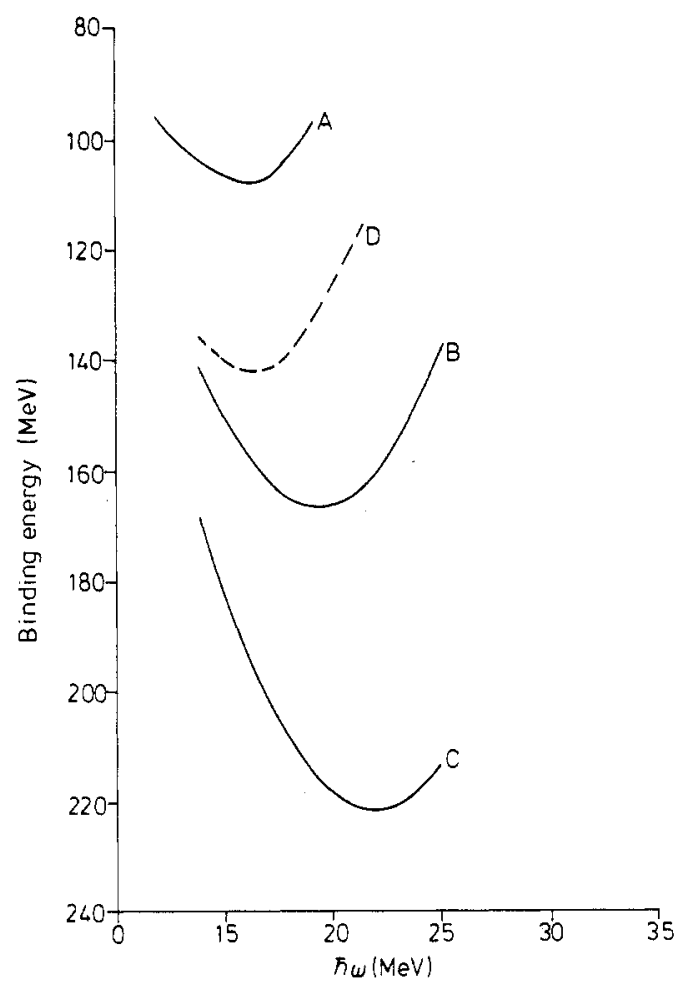

Figure 4. As figure 1 for ${ }^{16} \mathrm{O}$. In this case the broken curve (D), which includes explicitly the effects of the $\mathrm{N}^{*}(1234)$ and which yields saturation at the experimental binding energy, corresponds to $k=0.4$.

binding energy would reproduce the experimental binding, i.e. for ${ }^{4} \mathrm{He}$ with $k=0.2$, for ${ }^{12} \mathrm{C}$ with $k=0.42$ and for ${ }^{16} \mathrm{O}$ with $k \simeq 0.25$. The corresponding saturation densities, however, are too compact as expected.

When the effect of the $N^{*}$ 's is explicitly allowed for we find that the value of the constraint parameter which will yield the experimental binding energy is increased, and indeed in the case of ${ }^{12} \mathrm{C}$ it is increased to an unphysical size without significantly decreasing the nuclear saturation density.

\subsection{The normalisation constraint}

With the normalisation constraint most uncertainty surrounds a sensible choice of the constraint parameter $\bar{k}$. In the homogeneous fluid $\bar{k}$ is simply the Fermi momentum and is dictated by the density. We have thus related $\bar{k}$ to the density of the nucleus in question. We have used $\bar{k}$ to define an effective density $\bar{n}$

$$
\bar{n}=\frac{2}{3} \frac{k^{3}}{\pi^{2}}(2)^{-3 / 2}
$$

where the (2) $)^{-3 / 2}$ comes from our definition of the relative coordinate (equation (25)). The central density of a nucleus in the oscillator model is

$$
n_{0}=\sum_{i} 2\left(2 j_{i}+1\right)\left|\phi_{n_{i} i_{i} j_{i}}(h \omega, r=0)\right|^{2}
$$

and we have then considered constraints $\bar{k}$ corresponding to a range of values $\rho=\bar{n} / n$. 


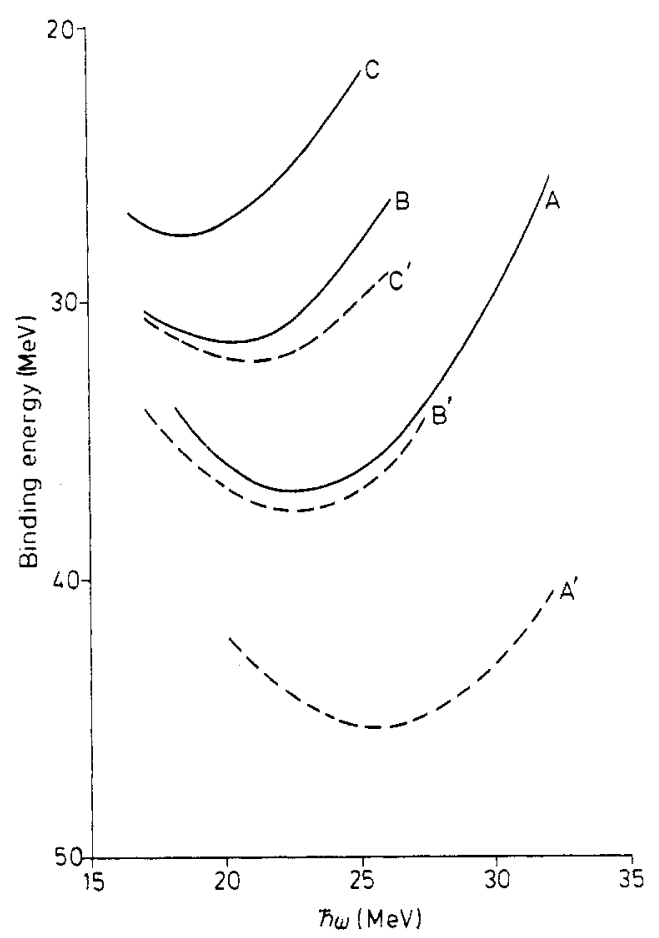

Figure 5. The full curves represent the results of LoCV calculations using the normalisation constraint with $\rho=(A) 0.35$, (B) 0.53 and (C) 0.71 for ${ }^{4} \mathrm{He}$. The broken curves $\left(\mathrm{A}^{\prime}-\mathrm{C}^{\prime}\right)$ are the corresponding results allowing for the $\mathrm{N}^{*}(1234)$ 's explicitly.

In figures 5, 6 and 7 we present the results of our calculations on ${ }^{4} \mathrm{He},{ }^{12} \mathrm{C}$ and ${ }^{16} \mathrm{O}$ respectively using the normalisation constraint and various values of $\rho$. We might have expected a reasonable choice of $\rho$ to be $\lesssim 1$ since the mean effective density inside the nucleus is $\leqslant n_{0}$.

We see that it is possible to choose values of $\rho$ such that the LOCV calculations reproduce the experimental binding energies (less Coulomb energy) and that this

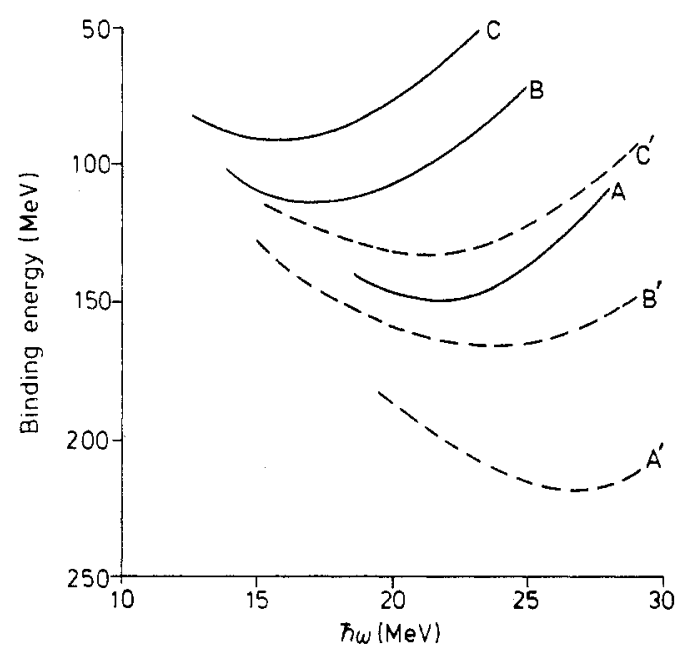

Figure 6. As figure 5 for ${ }^{12} \mathrm{C}$. 


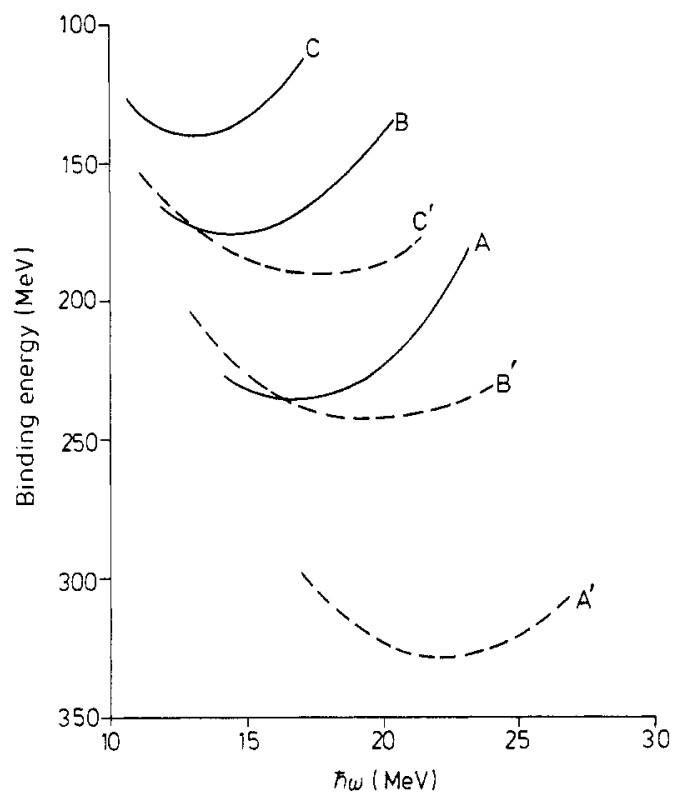

Figure 7. As figure 5 for ${ }^{16} \mathrm{O}$ but with $\rho=$ (A) 0.71 , (B) 1.06 and (C) 1.41 .

value of $\rho$ is an increasing function of mass. Thus we reproduce the experimental binding energy of ${ }^{4} \mathrm{He}$ with $\rho \simeq 0.71$ at $h \omega \simeq 21 \mathrm{MeV}$, of ${ }^{12} \mathrm{C}$ with $\rho \simeq 0.88$ at $\hbar \omega \simeq 19 \mathrm{MeV}$ and of ${ }^{16} \mathrm{O}$ with $\rho \simeq 1.77$ at $\hbar \omega \simeq 17 \mathrm{MeV}$. If we make explicit allowance for the $N^{*}(1234)$ 's effects on saturation, we see that it is again possible to choose values of $\rho$ which will reproduce the experimental binding energies. This occurs for ${ }^{4} \mathrm{He}$ with $\rho \simeq 0.53$ at $\hbar \omega \simeq 21 \mathrm{MeV}$, for ${ }^{12} \mathrm{C}$ with $\rho \simeq 0.57$ at $\hbar \omega \simeq 17 \mathrm{MeV}$ and for ${ }^{16} \mathrm{O}$ with $\rho \simeq 1.41$ at $\hbar \omega \simeq 13 \mathrm{MeV}$. While both calculations are capable of yielding the experimental binding energy and one might be led to believe that the effects of the $\mathrm{N}^{*}$ could simply be accommodated in a minor modification of the two-body correlation function, we see more clearly the saturating effects of the $\mathrm{N}^{*} \mathrm{~s}$ when we look at the densities. In table 1 we compare the calculated saturation RMS radii with experimental values. While explicit allowance for the $\mathrm{N}^{*}$ s leads to improved RMS radii, we do not feel we can make a detailed investigation of the nuclear density due to our approximation of using an oscillator basis rather than the self-consistent solutions of equation (21).

Table 1. Root mean square radii (fm).

\begin{tabular}{llll}
\hline Nucleus & LOCV & LOCV $+\mathrm{N}^{*}$ & Expt \\
\hline${ }^{4} \mathrm{He}$ & 1.7 & 1.7 & 1.63 \\
${ }^{12} \mathrm{C}$ & $2 \cdot 2$ & 2.4 & 2.47 \\
${ }^{16} \mathrm{O}$ & 2.4 & 2.7 & 2.65 \\
\hline
\end{tabular}

\section{Conclusions}

We have extended our LOCV calculations to a study of the ground states of closed-shell nuclei. We find that with the Bose constraint the results are extremely sensitive to 
the constraint parameter $k$ (equation (39)). Saturation can be obtained at the experimental binding energy but only at the expense of uncomfortably large values of $k(>0 \cdot 2)$. Such saturation generally corresponds to a too compressed nucleus. When explicit allowance for the saturation effects of the $N^{*}$ is made, the value of $k$ corresponding to the experimental binding energy increases sharply while the saturation density remains too compact. We would conclude that the Locv calculations for finite nucleus using the Bose constraint are unsatisfactory, there being no a priori way of selecting the constraint parameter, and when it is chosen to reproduce the observed binding it yields a too compressed density distribution and is sufficiently large as to cast doubts on the satisfactory convergence of the cluster expansion.

Turning to calculations with the normalisation constraint, there are now, in principle, no free parameters. However, the correlation function is now a function not only of the relative separation $r$ but also depends on the centre-of-mass coordinate $\boldsymbol{R}$ of the interacting pair of particles. Strictly, we should solve the Euler-Lagrange equations with the boundary condition that $f(r, R)$ heal to the function $\bar{h}$ of equation (40), or we might have made the local density approximation of equation (41). Instead, we have for simplicity chosen to ignore the dependence of $f$ on $R$ and we have assumed that all correlation functions heal to $h_{\text {eff }}$ of (42), corresponding to an effective average density given by $\bar{k}$ through equation (54). The results are extremely sensitive to the value of $\bar{k}$. When $\bar{k}$ is chosen to reproduce the observed binding energy it corresponds to a too compressed nucleus. If explicit allowance for the $N^{*}$ is included, the observed binding energy is reproduced with a smaller value of $\bar{k}$ and saturation occurs at an improved value of the RMS radii. These results are sufficiently encouraging to suggest that a more detailed investigation of LOCV techniques with the normalisation constraint applied to light nuclei is worthwhile.

The sensitivity of our results to the parameter $\bar{k}$ suggests that our assumption that the correlation functions can be made independent of the centre of mass of the pair of particles concerned is inadequate. Dropping this assumption leads to a considerable increase in the computational complexity of the problem, not only because the Euler-Lagrange equations have now to be solved at several values of the centre-of-mass coordinate but the equations themselves are more complicated due to the additional centre-of-mass dependence introduced into equation (27). Such calculations, healing to the function $\bar{h}$ of equation (40), would, however, be genuinely parameter free. Such calculations are in progress and will be reported on when complete.

\section{Acknowledgments}

We wish to acknowledge helpful comments from Professor A M Green on the role of the $\mathrm{N}^{*}(1234)$ in the saturation properties of nuclei. One of us $(\mathrm{CH})$ wishes to acknowledge receipt of a SRC Research Studentship Grant during the period of this work.

\section{References}

Brody $T$ and Moshinsky M 1960 Tables of Transformation Brackets (Mexico: Instituto de Fisica) Howes C, Bishop R F and Irvine J M 1978a J. Phys. G: Nucl. Phys. 4 L89

- 1978b J. Phys. G: Nucl. Phys. 4 L123 
$-1978 \mathrm{c}$ to be published

-1978d J. Phys. G: Nucl. Phys. 4 L45

Irvine J M et al 1976 Ann. Phys., NY 102129

Modarres M, Irvine J M and Bishop R F 1978 J. Phys. G: Nucl. Phys. 4 L127

Mueller G P and Clark J W 1970 Nucl. Phys. A 155561

Owen J C, Bishop R F and Irvine J M 1975 Ph's. Lett. 59B 1

-1976a Ann. Phys., NY 102170

-1976b Nucl. Phys. A 274108

-1977 Nucl. Phys. A 27745

Pandharipande V R 1971 Nucl. Phys. A 174641

_-1972 Nucl. Phys. A 18133

Ristig M L, TerLouw W J and Clark J W 1971 Phys, Rev. C 31504 